\title{
The decline in irradiation from the white dwarf in old novae
}

\author{
N. L. Thomas ${ }^{1}$, T. Naylor ${ }^{2}$, and A. J. Norton ${ }^{1}$ \\ 1 Department of Physics and Astronomy, The Open University, Milton Keynes, MK7 6AA, UK \\ 2 School of Physics, University of Exeter, Stocker Road, Exeter, EX4 4QL \& Astrophysics Group, Keele University, \\ Keele, Staffordshire, ST5 5BG, UK
}

Received 28 November 2007 / Accepted 18 March 2008

\begin{abstract}
Aims. We have investigated how a flux ratio analysis of the light curves of cataclysmic variables can be used to calculate the luminosity irradiating the secondary star in the classical novae QU Vul, V Per, DD Cir, DN Gem, V1432 Aql, and WY Sge.

Methods. We undertook phase-resolved, near-infrared $K$ band photometry of QU Vul and V Per. Using data from QU Vul we show how flux ratios taken between fiducial orbital phases in the light curves of irradiated CVs can be used to measure the degree of heating of the secondary star. We compared the heating effect obtained from flux ratio analysis with more formal modelling, or by measurements taken from the literature, and found good agreement. We used the results to determine how irradiation changes with time since the nova outburst.

Results. The light curve of QU Vul shows the presence of two maxima in the $K$ band, which are displaced from phase 0.25 towards the 0.5 phase position, as would be expected from heating of the inner face of the secondary star by radiation from hot primary. Nova V Per, on the other hand shows evidence for a hot spot on the accretion disc, and it would appear that heating of the inner face is not occurring. The results of the flux ratio analysis of the objects examined are plotted as a function of time since the nova explosions occurred. There is marginal evidence for a decline in flux with time since the outburst, superimposed on considerable scatter, which is likely to be caused by the different temperature reached in each nova explosion. The decline is consistent with the declines others have seen. We conclude that it is the decline in reprocessed irradiation from the cooling white dwarf alone, rather than a decline in mass transfer rate, that could be the cause of the decrease in optical brightness seen in old novae.
\end{abstract}

Key words. stars: novae, cataclysmic variables - stars: white dwarfs

\section{Introduction}

Cataclysmic variables (CVs) is the term given to the group of objects which are semi-detached interacting binary stars composed of a white dwarf primary star and a low mass $\left(<0.5 M_{\odot}\right)$, normally main sequence, secondary star with orbital periods of typically less than 1 day. Observations have led to a classification scheme for CVs, which includes dwarf novae, recurrent novae, novalike variables, and magnetic CVs. In each category, the secondary star loses mass via Roche-lobe overflow to the primary, the strength of the magnetic field of the white dwarf determining whether mass transfer of hydrogen-rich gas occurs via an accretion disc or whether it is channelled directly onto the surface of the white dwarf.The observed luminosity of the non-magnetic systems arise from three components; the white dwarf, the secondary star, and the accretion disc which dominates the visual flux.

Classical novae are CVs that have been observed to undergo brightness increases of 6-15 mag, releasing $10^{44}$ to $10^{46} \mathrm{erg}$, which are believed to be due to thermonuclear runaways on the surface of the white dwarfs. For a review see Warner (1995). The material for the runaway is supplied by accretion onto the white dwarf, from the late-type low-mass star in orbit around it. By definition only one such outburst has been observed for each classical nova. The overall structure of these systems is observationally indistinguishable from other CVs and theories have been constructed, for example by Patterson (1984) and Downes (1986), which suggest that any CV will undergo a nova explosion every ten thousand years to a million years. During the nova explosion the white dwarf rapidly increases in temperature, and theoretical work by Prialnik (1986), modelling the evolution of classical novae, predicts that the white dwarf itself will remain hot from the nova explosion for many years. The resulting "hot" white dwarf subsequently irradiates the front face of the secondary star and any accretion disc that may have formed. Both the irradiated disc and the heated secondary star give rise to an increase in the system luminosity with the disc flux being the more dominant of the two.

Shara et al. (1986), in proposing their hibernation theory to explain the discrepancy between nova outburst frequencies (both observed and theoretical) and the space density of classical nova in our Galaxy, noted that old novae are observed to remain bright for a century after eruption, and concluded that this was usually due to enhanced mass transfer from the red dwarf to the white dwarf. They suggested that the effect of the irradiation on a star with a deep convective envelope, as in CV secondaries, is to block a portion of the luminosity, reducing the effective surface luminosity causing the star to swell. As the star swells, Roche lobe overflow occurs driving an enhanced mass transfer rate. Work undertaken by Kovetz et al. (1988) appeared to lend support for this hypothesis when simulation of time-dependant irradiation of three red dwarf models showed that the irradiation caused the red dwarf outer layers to expand by up to $2 \%$ and overflow the star's Roche lobe surface, enhancing mass transfer rates by two orders of magnitude.

Thus in addition to the white dwarf directly increasing the luminosity of the disc and secondary, the irradiated secondary may expand and so lead to an increase in the mass transfer rate, 
which in turn will increase the disc luminosity to a greater extent than that due solely to irradiation of the disc alone.

As the white dwarf cools over a few centuries, the irradiation must lessen and the luminosity of the irradiated disc and secondary will decline. Furthermore, as the irradiation decreases so does the red dwarf's expansion. As a result, mass transfer $\dot{M}$ declines with time $t$ as $\dot{M} \propto t^{-3}$ which in turn causes a further decline in the disc luminosity. Prialnik (1986) showed that the luminosity $L$ due to cooling and contraction of a white dwarf after a nova explosion follows a decline with time $t$ in which $L \propto t^{-1.14}$. Somers \& Naylor (1999) confirmed that the cooling rate of the white dwarf in V1500 Cyg is consistent with the model of Prialnik.

In undertaking a statistical analysis of nova eruption amplitudes based on a sample of 97 well observed galactic novae, Vogt (1990) determined that, during the 130 years after eruption, these systems showed a decrease in brightness with a average rate of $2.1 \pm 0.6 \mathrm{mag}$ per century and interpreted the result in terms of a secular decline in the mass transfer rate. In addition, Duerbeck (1992) analysed a series of photoelectric observations for each of 9 old novae and determined a mean rate of decline of $10 \pm 3$ millimag per year half a century after outburst, and also interpreted them in the context of the hibernation scenario, as a decrease in mass transfer from the secondary star.

However, Weight et al. (1994) carried out near infrared photometry of a number of old classical novae and related objects, and used the data to estimate the mass transfer rate in these systems. They found no correlation between mass transfer rate and the time since outburst, at least over the 140 year period for which they had data, and no evidence to support Vogt's (1990) conclusion that the mass transfer in a classical nova system declines after outburst. They concluded that if novae do enter a phase of hibernation, then the mass transfer cannot begin to decline until at least 140 years after outburst.

Schreiber \& Gänsicke (2001) in presenting detailed calculations of irradiated discs in $\mathrm{CVs}$, showed that the decreasing irradiation from the white dwarf necessarily implies a significant decline of the visual brightness in post novae due soley to changes in the disc. Their results are found to be in agreement with the declines in post novae found by Duerbeck, and they concluded that the decrease of disc irradiation by the cooling white dwarf in post novae results in a decrease of their visual flux. Even though a decrease in mass transfer rate may contribute to the observed long term visual decline in post novae, the effect of decreasing disc irradiation alone is sufficient to explain the observed decline rate.

\section{Aims of this work}

The object of this study is to establish whether information from the lightcurves of eclipsing old novae can be used to determine the degree of the heating effect, either by formal lightcurve modelling or by simpler flux ratio measurements. Using these measures of the heating effect, the aim is then to see how irradiation declines with the time since the nova explosion and test whether the decline in brightness is due to a reduction in the luminosity of the irradiated secondary and accretion disc or due to a reduction of the expansion of the secondary causing a decrease the mass transfer rate, which in turn, leads to a fall in the disc luminosity.

For an unheated, Roche-lobe filling star, the orbital light curve shows two minima per cycle when the inner and outer Lagrangian points are towards the observer. These modulations, known as ellipsoidal flux variations (or more correctly Roche distortional variations), are caused by the distorted shape of the

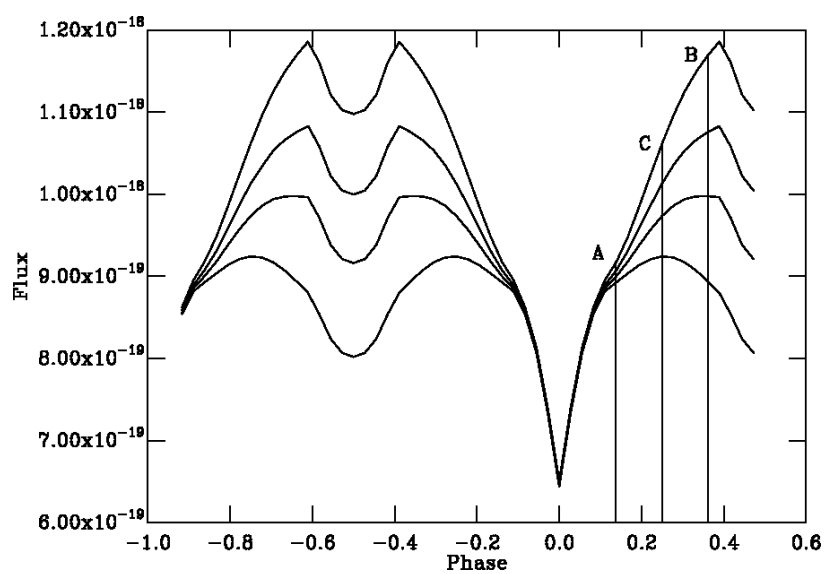

Fig. 1. Flux as function of phase in a typical eclipsing CV, showing the effect of a change in the irradiated flux. The model curves (from top to bottom) are for $1.8 L_{\odot}, 0.9 L_{\odot}, 0.4 L_{\odot}$, and $0.005 L_{\odot}$ respectively. The flux units are erg cm $\mathrm{cm}^{-2} \mathrm{~s}^{-1} \AA^{-1}$. Fiducial phase points A, B, and C are explained in Sect. 8.

secondary star in a binary system, because of the tidal forces exerted by the primary white dwarf. The result is a change of the surface area of the companion, seen by the observer at different phases of the cycle. Such light curves, for example those shown by Paneck \& Eaton (1982) in U Gem, typically have two maxima at phases 0.25 and 0.75 relative to conjugation.

The effect of increasing the WD temperature in a typical eclipsing $\mathrm{CV}$ can be demonstrated by using modelling such at that described in Sect. 8.0 and is shown in Fig. 1. As the irradiated flux from the WD is increased, the temperature of the irradiated area on the secondary increases and the maximum flux in the direction of the observer is seen at progressively later phases as the contribution from the ellipsoidal flux becomes dominated by the the irradiated surface flux. Thus heating the inner Lagrangian face has the effect of filling in the secondary minimum, so that the maxima (normally at phase 0.25 and 0.75 ) move towards phase 0.5 . This effect has been observed by Somers et al. (1996) in WY Sge, and was used to prove that the heating of the secondary star by its white dwarf is small. By measuring the flux at fiducial phases $\mathrm{A}, \mathrm{B}$, and $\mathrm{C}$ on the lightcurve, we show how a relative measure of the degree of heating of the secondary can be determined and then compare the results obtained with this technique with that of more formal modelling.

Nova Vulpecula 1984 (QU Vul) is an ideal candidate with which to investigate the effect of heating since it is a recent nova, and hence has a hot white dwarf, that shows eclipses and thus has a constrained inclination. In addition a second nova, V Per, which erupted in 1887, was observed to examine the effects of cooling over a 110 year period. We also took data from the literature for other systems having a heated secondary star, DD Cir, DN Gem, V1432 Aql, and WY Sge and show how the irradiation effect declines with time

\section{The target stars}

The classical nova QU Vul was discovered on December 22.13 UT 1984 and although it became one of the brightest and best studied recent novae reaching $V=5.6$ there is, at present, a lack of data on the binary system parameters. QU Vul belongs to the group of novae in which the seat of the outburst is a thermonuclear runaway occurring on 
Table 1. Journal of observations for QU Vul and V Per.

\begin{tabular}{ccccc}
\hline \hline Date & Object & Exposure (s) & Filter & No. of images \\
\hline 16 July 1997 & QU Vul & 10 & $K$ & 956 \\
17 July 1997 & V Per & 10 & $K$ & 415 \\
\hline
\end{tabular}

a ONeMg white dwarf. Shafter et al. (1995) undertook time resolved photometric and spectroscopic observations which established that QU Vul undergoes eclipses (0.5 mag deep in the $V$ band) and that it has an orbital period of $2.68 \mathrm{~h}$. The ephemeris of QU Vul was recalculated using our observations and the data in Shafter \& Misselt (1995) as $T_{\text {mid-eclipse }}=$ BJD 2.450080.523(4) $+0.1117648(8) E$ with a reduced $\chi^{2}$ of 20.8 and an RMS O-C of $1.88 \times 10^{-2}$ days in close agreement with that of Shafter et al.

V Per, discovered on 3 November 1887, is a cataclysmic variable with a quiescent visual magnitude of approximately 18 . Again there is little known about the basic parameters of this binary system. Shafter \& Abbott (1989) discovered that the system eclipses with a period of $2.57 \mathrm{~h}$. The eclipses are approximately 1.3 mag deep, in red light, having a V shape with a full width at half intensity of approximately $11 \mathrm{~min}$. Shafter \& Abbott noted that the light curve displays a steep ingress and egress without any obvious evidence of a differentiated white dwarf eclipse, typical of nova like systems with a high mass accretion rate and optically thick discs. Shafter (1997) estimated that V Per lies at distance of $1.0 \mathrm{kpc}$.

\section{Observations}

Infrared $K$ band $(2.182 \mu \mathrm{m})$ observations of QU Vul and V Per were taken with the $3.8 \mathrm{~m}$ United Kingdom Infrared Telescope (UKIRT) at Mauna Kea using the IRCAM3 at the Cassegrain focus on 16 and 17 July 1997, as shown in Table 1. The plate scale was $0.286 \mathrm{arsec} / \mathrm{pixel}$. The seeing for QU Vul on 16 July, given by the geometric mean of the maximum and minimum width of an elliptical Gausian fitted to the object stars PSF function, varied during the observations between 1.1 and 1.7 arcsec at the beginning of the observing run to between 0.6 and 0.9 at the end. On 17 July seeing was relatively constant between 0.9 and 1.4 arcsec for the V Per observations.

The raw images were taken in groups of 8 data frames. After each frame the telescope was moved by a small amount in RA and Dec. This causes the star of interest to fall on different parts of the array. The telescope motion is cyclic, and after one cycle the star of interest falls on roughly the same part of the array as in the first exposure. This exposure then marks the beginning of the next cycle, and the telescope repeats the same interexposure movements. The reason for observing in this manner is that in each cycle of the telescope motion, any particular pixel will have been illuminated by the sky in at least one frame. This allows the sky to be used as a source of uniform illumination with which to remove the pixel to pixel sensitivity variations of the array.

\section{Data reduction and analysis}

Each image was dark subtracted and then flat-fielded with the median of all the frames in the group, excluding itself. The times of mid exposure were corrected to Barycentric Dynamical Julian Date. Photometry was carried out using the optimal extraction technique developed by Naylor (1998). We removed atmospheric extinction variations by means of differential photometry using several bright stars in the image frame. The magnitudes

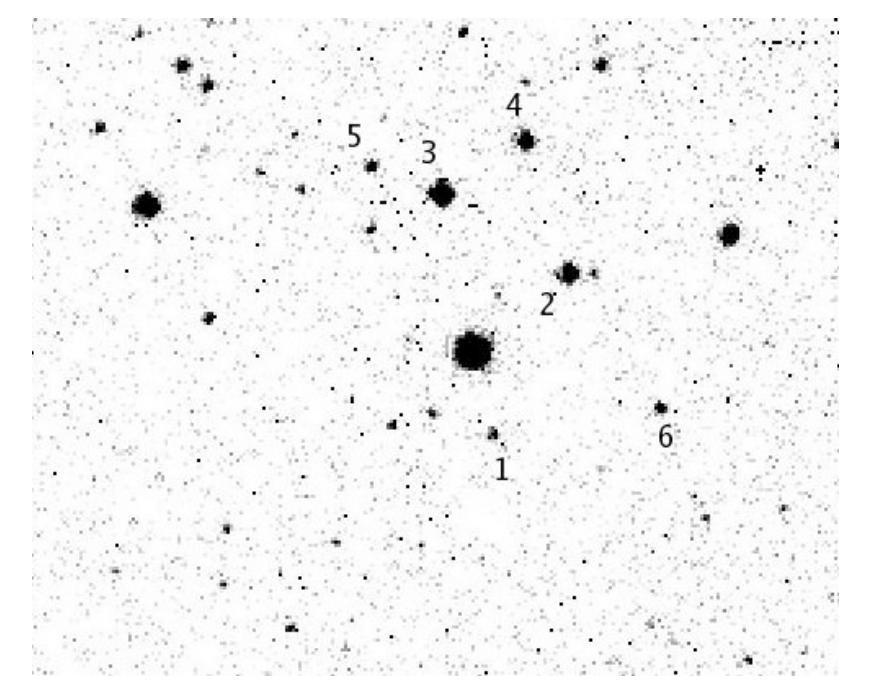

Fig. 2. The field around QU Vul in the $K$ band. The field of view is approx $73 \times 73$ arcsec. Star1 is the nova. Its identification is taken from Shin et al. (1998). North is at the top and East is to the left of the frame.

Table 2. Equinox J2000 co-ordinates of the comparison stars used in the photometry for QU Vul observations together with their observed $K$ band magnitudes.

\begin{tabular}{ccccc}
\hline \hline Star & RA & Dec & $\begin{array}{c}\text { Calculated } \\
K \text { Mag }\end{array}$ & $\begin{array}{c}\text { Calculated } \\
K \text { Mag } \\
\text { uncertainty }\end{array}$ \\
\hline Star2 & $20: 26: 45.46$ & $+27: 50: 56.9$ & 13.965 & 0.015 \\
Star3 & $20: 26: 46.28$ & $+27: 51: 04.6$ & 13.294 & 0.010 \\
Star4 & $20: 26: 45.77$ & $+27: 50: 09.1$ & 13.294 & 0.010 \\
Star5 & $20: 26: 46.78$ & $+27: 51: 07.4$ & 15.388 & 0.050 \\
Star6 & $20: 26: 44.92$ & $+27: 51: 43.9$ & & \\
\hline
\end{tabular}

of the target stars and the surrounding field stars in each frame were calibrated by photometry of the UKIRT Faint Standard stars FS29, FS32, and FS35, as listed by Hawarden et al (2001), taken during the same observation run. Standard star calibrations were determined to a mean uncertainty of 0.007 mag with an uncertainty in the mean of 0.003 for $K$ band stars. Figure 2 shows the field around QU Vul. The Equinox J2000 co-ordinates of the comparison stars used in the photometry of QU Vul taken from the 2MASS Point Source Catalogue All Sky Survey 2003 by Cutri et al. (2003) together with the results of our photometry are shown in Table 2. Figure 3 shows the field around V Per. The Equinox J2000 co-ordinates of the comparison stars used, again taken from 2MASS, and the $K$ band magnitudes of these stars, obtained from the photometry, are shown in Table 3.

\section{Results of photometry for QU Vul}

Figure 4 shows the resulting $K$ band light curves for QU Vul after binning the data into periods of approximately $8 \mathrm{~min}$ using a weighted mean. Despite gaps in the data (when the telescope was switching to other filters or targets) the light curve shows a distinct maximum at phase 0.4 and a deep minimum at phase 0 and also gives a hint of an eclipse of the secondary star by the disc/white dwarf at phase 0.5 .

In addition, a steady decline in the brightness of the maximum and minimum is also apparent. There is no fall in the trend of the light curve of the neighbouring field stars or that of a blank sky region, and the result is the same whether optimally weighted photometry or simple aperture photometry is used, 


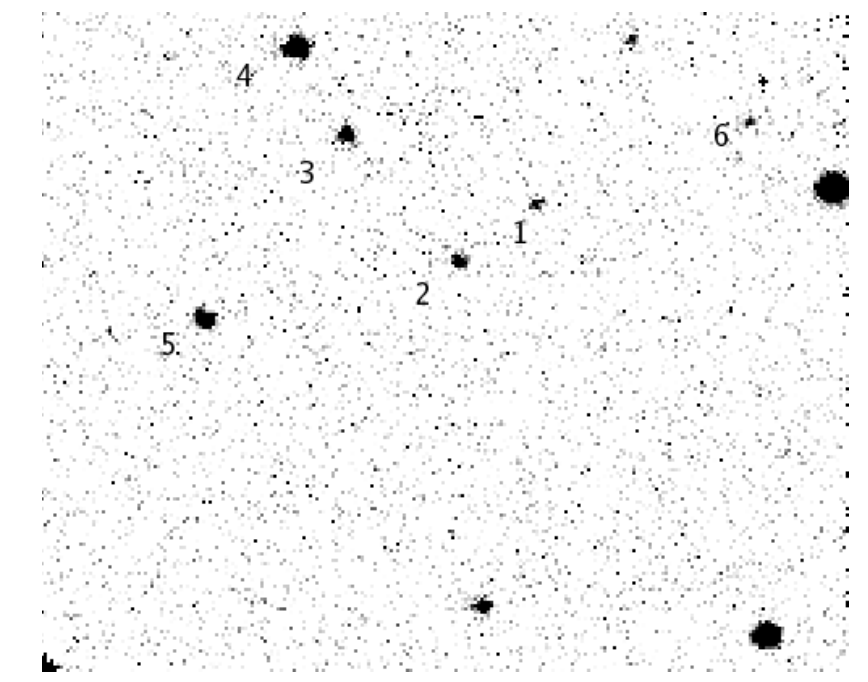

Fig. 3. Field around V Per in the $K$ band. The field of view is approx $73 \times 73$ arcsec. Star1 is the nova its identification is taken from Downes et al. Catalogue of Cataclysmic variables $(+2001-2005)$. North is at the top and East is to the left of the frame.

Table 3. Equinox J2000 co-ordinates of the comparison stars used in the photometry for V Per observations together with their observed $K$ band magnitudes.

\begin{tabular}{ccccc}
\hline \hline Star & RA & Dec & $\begin{array}{c}\text { Calculated } \\
K \text { Mag }\end{array}$ & $\begin{array}{c}\text { Calculated } \\
K \text { Mag } \\
\text { uncertainty }\end{array}$ \\
\hline Star2 & $02: 01: 54.74$ & $+56: 43: 58.7$ & 15.707 & 0.052 \\
Star3 & $02: 01: 55.98$ & $+56: 44: 10.4$ & 15.171 & 0.033 \\
Star4 & $02: 01: 56.38$ & $+56: 44: 17.9$ & 14.075 & 0.015 \\
Star5 & $02: 01: 57.47$ & $+56: 43: 54.2$ & 14.942 & 0.018 \\
Star6 & $20: 01: 52.95$ & $+56: 44: 18.2$ & 16.398 & 0.094 \\
\hline
\end{tabular}

demonstrating that the decline seen in the QU Vul light curve is real and not an effect of the data reduction process. While the flux level at phase zero falls by approximately $\Delta m=0.6 \mathrm{mag}$ over the two and a half $2.68 \mathrm{~h}$ orbital periods observed during the run, the maximum amplitude of each cycle (defined as flux level at peak minus flux level in primary eclipse) is steadily increasing by $25 \%$ in the second cycle and $30 \%$ in the third cycle.

The cause of steady decline in the flux is not certain but may well be associated with the repetitive optical brightening seen in some post novae. Hoard et al. (2000) has observed a variability of $\Delta V$ mag 0.4 to 0.8 on time scales of 30 to 50 days for DI Lacertae and V841 Ophiuchi, while Warner (1995) notes similar variations in the light curves of other nova remnants. The physical origin of these variations on such time scales has not yet been identified but may be related to disc instabilities or mass transfer from the secondary star.

It is clear from the $K$ band light curve that the secondary star in QU Vul is being irradiated, ellipsoidal modulation is absent, and the system is dominated by the inner face heating.

\section{Results of photometry for V Per}

The $K$ band light curve for $\mathrm{V}$ Per (Fig. 5) shows a primary eclipse at phase 0 of just under 1 mag. Maximum light from the system occurs at phase 0.75 as would be expected by purely ellipsoidal modulation when seeing the distorted secondary star side on. However, there is no corresponding maximum at phase 0.25 , suggesting an alternative interpretation as

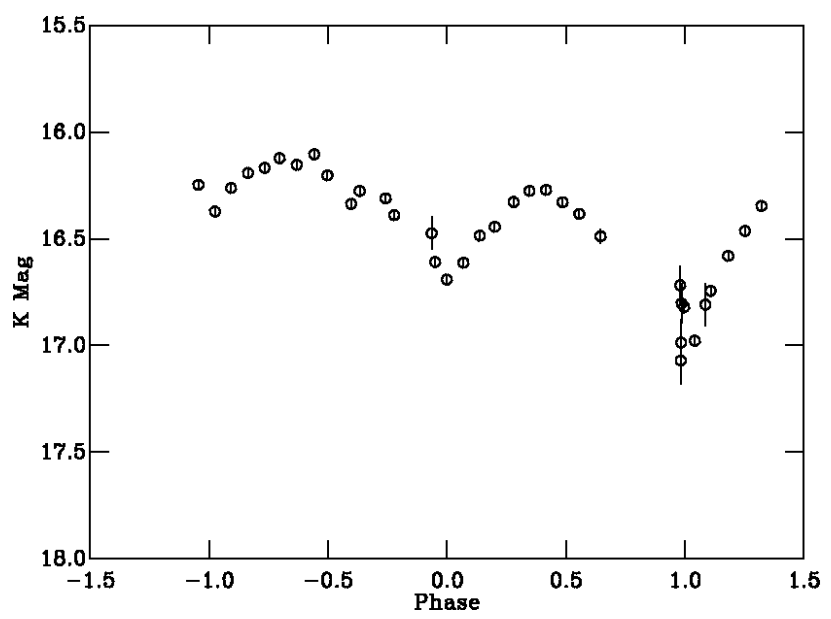

Fig. 4. QU Vul $K$ band light curve, binned into approximately $8 \mathrm{~min}$ samples using the weighted mean errors derived from the photometry, showing the overall fall in lumininosity of the system. The vertical bars are overall uncertainty.

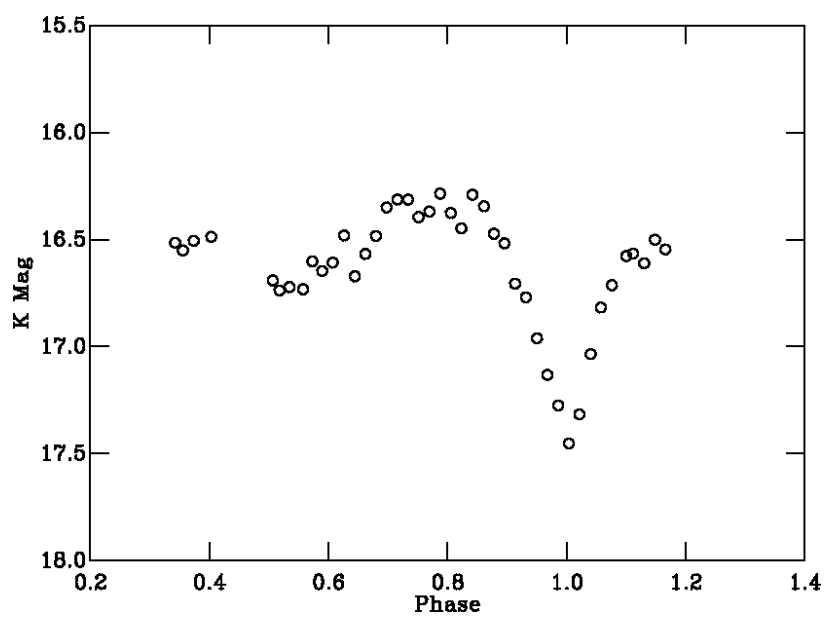

Fig. 5. V Per light curve binned into approximatley 2.5 min samples using the weighted mean errors derived from photometry.

a bright spot. Support for this view comes from observations in other wavelengths. Although not visible in observations using a broad-band $B V R$ filter transmitting between 4000 to $7000 \AA$ Shafter \& Abbott (1989), using a RG 610 filter (red response blocking light below $\approx 6100 \AA$ ), produced a light curve for V Per in which the presence of an orbital hump just prior to inferior conjunction of the red star appears to have been detected. In the $K$ band observations this hump is very prominent.

\section{Irradiation of secondary stars by hot white dwarfs}

QU Vul clearly shows signs of a heated secondary star whereas V Per does not appear to be significantly irradiated. The extent of the irradiation in QU Vul and V Per, and four other CVs which clearly show signs of a heated secondary star (DD Cir, DN Gem, WY Sge and V1432 Aql) can be determined by flux ratio analysis of their light curves, and the resulting values compared with those obtained either from values taken from the literature or from modelling. This flux ratio technique is explained in Sect. 8.1 and then applied to QU Vul, V Per and other systems in Sects. 8.2 to 8.7 . 
Comparison of modelled ellipsoidal variations with observed light curves is a way of deducing the basic parameters of interacting binaries. This technique has been used successfully, for example by Shabaz et al. (1993), in investigating ellipsoidal variation of a low mass X-ray binary system Cen X-4, and by Somers et al. (1996) to provide constraints on the system parameters of WY Sge.

The model used here, developed by Shabaz et al. (1993), describes the cool component as a Roche lobe filling star, radiating with a blackbody flux distribution incorporating gravity darkening and approximate temperature-dependant limb darkening using coefficients taken from Al-Naimiy (1978). It is similar to ellipsoidal variation models computed by Mochnacki \& Doughty (1972), Bochkarev et al. (1979), Wilson \& Devinney (1979), Hill (1979) and Tjemkes et al. (1986). Somers et al. (1996) added to the model an optically thick accretion disc, flared so as to be triangular in radial cross section, having a blackbody flux distribution and a radial temperature distribution for a steady state disc. Ioannou et al. (1999) further modified the code to take into account the irradiation of the surface of the secondary star by a heating source situated at the centre of the accretion disc and added limb darkening coefficients for the accretion disc. The heating source term which governs the total irradiation from the WD together with any contribution from the accretion disc or boundary layer is set, in the model, by the white dwarf temperature. The mass transfer rate parameter simply controls the flux from the disc. The white dwarf and secondary star masses, white dwarf temperature, secondary star pole temperature, mass transfer rate, accretion disc inner and outer radius, disc opening angle, inclination, gravitational darkening limb darkening coefficients, and the distance to the CV are all independent parameters in the model. Results of fitting such models to our data are discussed in Sects. 8.2 and 8.5.

\subsection{Irradiation flux ratio analysis}

Evidence of irradiation of the secondary star by the white dwarf in many CVs can be seen their light curves and it should be possible to deduce the extent of reprocessed radiation from consideration of the light curve alone. The effect of increasing the irradiating flux on the secondary star in a CV is to change the morphology of the light curve.

We have investigated the effect of irradiation of the secondary star by the white dwarf by considering the flux at three points $\mathrm{A}, \mathrm{B}$, and $\mathrm{C}$ in the light curve in heated and unheated secondaries (see Fig. 1). We define phase A to occur just outside the primary eclipse, phase B to occur just outside the secondary eclipse, and phase $C$ to be 0.25 . Then, in an irradiated $C V$, the flux at phase A consists of light from the accretion disc plus the flux from the unheated side of the secondary star. The phase B flux consists of the flux from the accretion disc together with a large proportion of the flux from the heated face of the red star. At phase $\mathrm{C}$ flux from the disc and the maximum projected area of the secondary star is seen, the ellipsoidal component, together with a lower proportion of flux from the irradiated surface of the secondary star than is seen at phase B. In the case of an non irradiated $\mathrm{CV}$, phase A flux consists of light from the accretion disc plus the flux from the outer-Langrangian-point face of the secondary star. Phase B flux comes from the accretion disc together with flux from the inner-Langrangian-point face of the red star. At phase $C$ the flux from the ellipsoidal component is seen together with the flux from the accretion disc. Subtraction of the fluxes occurring at the phases $\mathrm{B}$ or $\mathrm{C}$ from the flux at a flux $\mathrm{A}$ (i.e. (Flux B-Flux A) or (Flux C-Flux A)) has the effect of

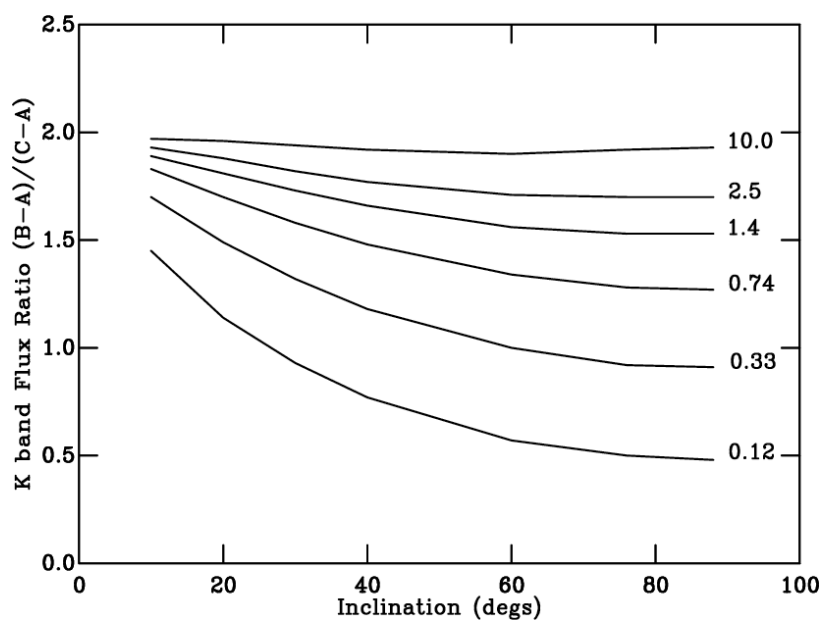

Fig. 6. $K$ band flux ratio as a function of inclination for varying WD luminosity $L_{\odot}$.

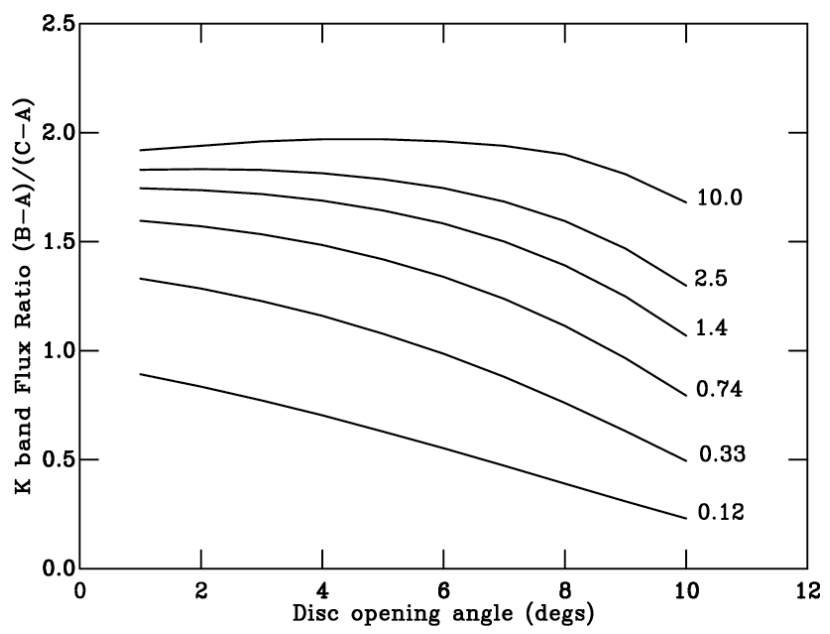

Fig. 7. $K$ band flux ratio as a function of disc opening angle for varying WD luminosity $L_{\odot}$.

subtracting out the flux from the accretion disc, leaving behind the difference in fluxes from the secondary star at the two phases.

In the case of a non-irradiated secondary, the difference in fluxes (Flux B-Flux A) approximates to the flux from the unheated inner-Langrangian-point face of the secondary star, while the flux difference (Flux C-Flux A) corresponds to the flux from the ellipsoidal component. The ratio of fluxes (Flux B-Flux A)/(Flux C-Flux A) should normally be $<1$. For an irradiated $\mathrm{CV}$ the difference in fluxes (Flux B-Flux A) approximates to the flux from the irradiated inner-Langrangian-point face of the secondary star, and the flux ratio (Flux B-Flux A)/(Flux C-Flux A) for the irradiated case is therefore expected to be $>1$.

In order to show the validity of this relationship we modelled a typical eclipsing $\mathrm{CV}$, varying the individual system parameters under a range of irradiating luminosities, and calculated the flux ratios. Figures $6-8$ show, for example, how the flux ratio varies as a function of inclination, disc opening angle and mass ratio for varying WD luminosity $L_{\odot}$. From modelling a given $\mathrm{CV}$, the relationship between flux ratio and irradiating luminosity at various wavelengths can be determined. The form of this relationship, which is independant of the CV system modelled, is shown in Fig. 9. 


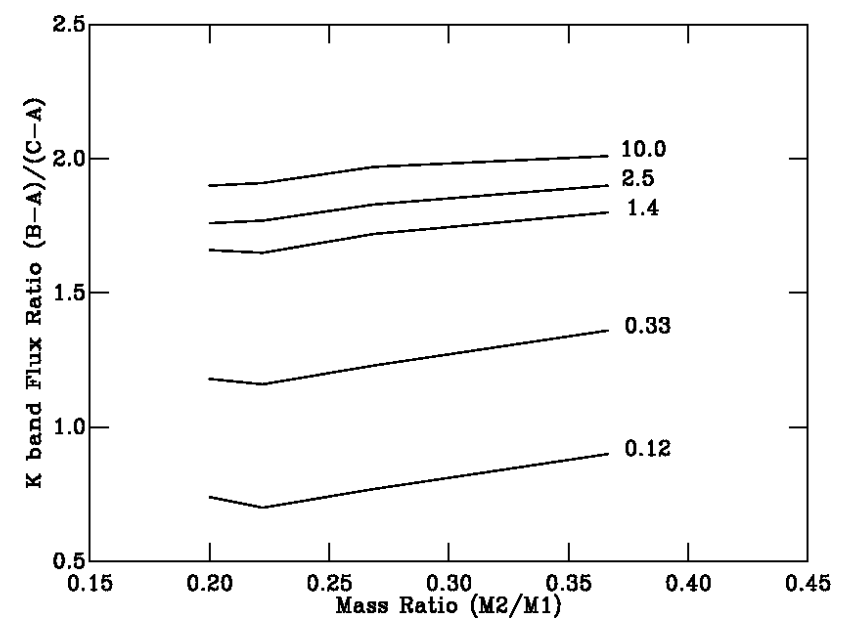

Fig. 8. $K$ band flux ratio as a function of mass ratio for varying WD luminosity $L_{\odot}$.

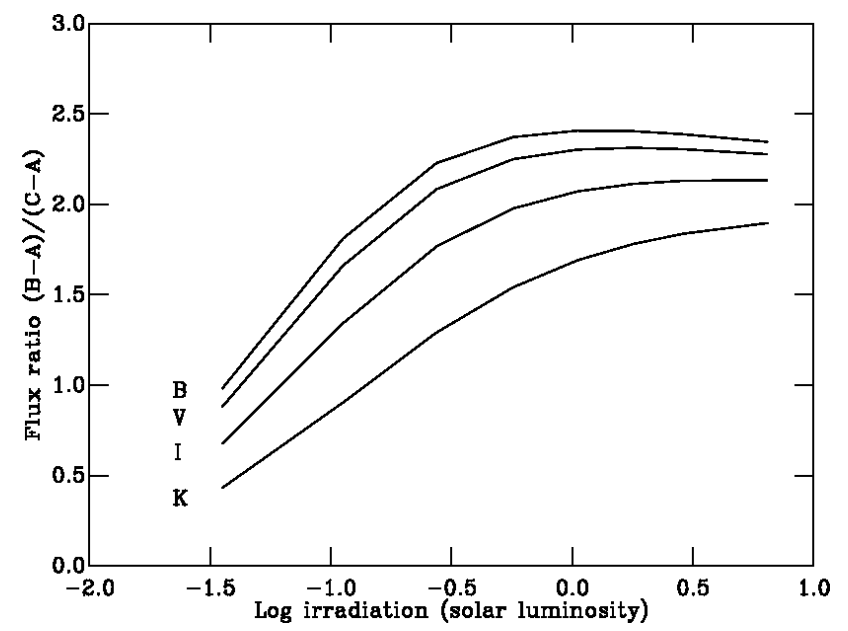

Fig. 9. Flux ratio as a function of $\log$ irradiation $L_{\odot}$ for varying wavelength.

Since we are dealing with eclipsing systems, we cannot observe the maximum irradiated surface area of the secondary star, and hence true maximum flux, which would appear at phase 0.5 as it is partially obscured by the disc. The technique used to derive the WD luminosity from the light curve is therefore limited by the maximum flux which can be observed at the fiducial points outside the eclipse regions. The models show that the maximum ratio that can be seen is approximately between 1.7 and 2.4 dependant upon the wavelenghth of observation. This corresponds to a maximum irradiation luminosity of approximately $1 L_{\odot}$ for short wavelength and approximately $6 L_{\odot}$ for long wavelengths. Thus the technique, when applied using infrared observations, can clearly distinguish between weak $\left(1 L_{\odot}\right)$ and strong irradiation.

The method is, however largely independent of such basic parameters as mass transfer rate, secondary star temperature, and hence distance to the system etc, which are quite often unknown in these systems. In addition since it relies on relative fluxes, absolute photometry of these systems is not required.

\subsection{QU Vul irradiation}

The effect of the steady decline in the amplitude of the maxima and minima in the QU Vul lightcurve was reduced by fitting a linear regression line to the raw lightcurve, subtracting the regression line from this light curve, and then normalising the resulting light curve about the mid-point of the regression line slope. Data points taken at the fiducial positions on the second orbital cycle of the regression corrected $K$ band light curve gave a mean flux ratio of 1.757 with a standard deviation of 0.056 . When converted to irradiating luminosity this gave a mean luminosity of $1.3 L_{\odot} \pm 0.3$ for QU Vul.

In order to verify the flux ratio results we undertook modelling of QU Vul. To successfully model the QU Vul light curve, the regression corrected light curve was binned into approximately 0.087 phase samples, enabling reasonable computational time, using a weighted mean with uncertainties taken from the photometry. Data used for modelling was restricted to that for the first half of the 2nd orbital cycle in order to further minimise the effects of the changing light curve.

For the purposes of modelling, the mass of the secondary star in QU Vul was assumed to be $0.22 M_{\odot}$ based on the mean empirical mass period relationship derived by Warner (1995) which uses the mass-density relationships for the secondaries in CVs obtained by Webbink (1990). QU Vul is a neon nova and, according to Ritter et al. (1991), it is believed that ONeMg white dwarfs tend to be massive stars. However estimates of the ejected mass of QU Vul by Saizar et al. (1992) and others are consistent with a white dwarf mass of $1 M_{\odot}$ or less, and so an assumed value for the mass of the primary of $0.82 M_{\odot}$ was taken from Webbink (1990), who derived masses of the white dwarf primaries in $\mathrm{CV}$ s from a variety of methods. A gravity-darkening coefficient of 0.8 was chosen as this is the theoretical value for a convective envelope for the secondary star, and limb-darkening values were taken from Al-Naimiy (1978).

Conflicting distance estimates for QU Vul have been obtained (see Gehrz 1985; Bergner et al 1985; Andrea et al. 1994; Della Valle et al. 1997; Taylor et al. 1998; and Downes \& Duerbeck 2000), ranging from Bergner et al. who concluded the distance to QU Vul was $1.16 \pm 0.02 \mathrm{kpc}$ to Taylor et al who obtained a distance of $3.6 \mathrm{kpc}$. For the purposes of our modelling we were looking for the minimum level of irradiation and so $1.1 \mathrm{kpc}$ was adopted in this paper.

Owing to the large number of free parameters in the model, no formal fitting of the light curve was undertaken. Reasonable initial values for each of the free parameters were assumed and the model was run repeatedly varying each parameter in turn to assess its relative effect on the light curve. Inclination and white dwarf temperature had by far the greatest effect on the shape of the model light curve, while the disc size (based on fractions of the distance from the centre of the primary to the inner Lagrangian point L1), mass ratio, and mass transfer rate had a less significant effect, and the remaining parameters the least. The white dwarf temperature was adjusted to make the maximum flux occur near phase 0.4 , and the angle of inclination was set to make the maximum flux level equal to that in the photometric curve. The crude fit was then further refined by carrying out grid searches between pairs of parameters to obtain the minimum $\chi^{2}$ goodness of fit between the model and the light curve, and the process repeated with new values for each parameter in turn until no further improvement could be made.

The results of the best fit modelling for the QU Vul $K$ band light curve superimposed on the $K$ band photometric curve are shown in Fig. 10 and the model parameters are shown in Table 4.

From the best-fit model for QU Vul, the temperature of the "hot white dwarf" is $65000 \mathrm{~K}$. At that temperature it radiates at approximately $1.4 L_{\odot}$. The model requires a mass transfer rate of $7 \times 10^{-9} M_{\odot} \mathrm{yr}^{-1}$ which if simply converted to accretion 


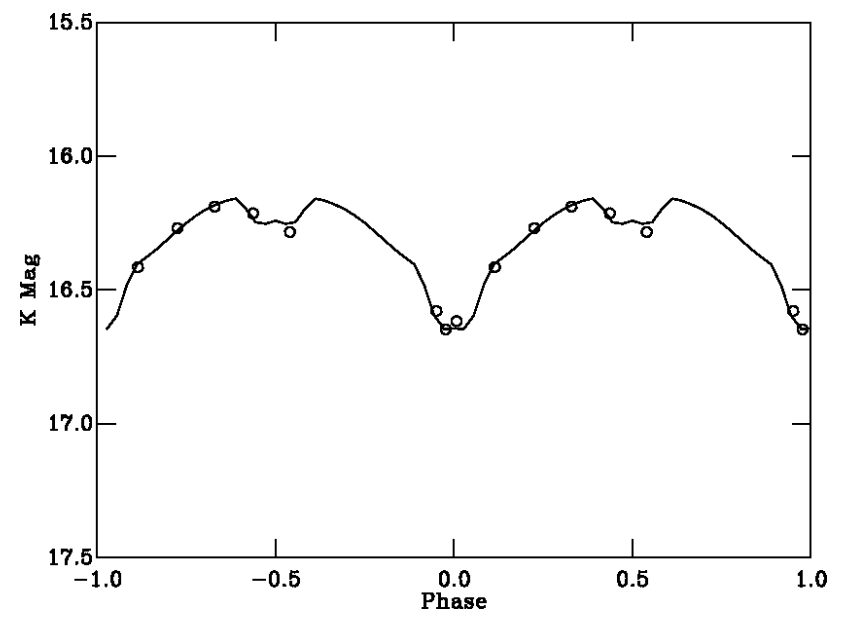

Fig. 10. Best fit model for the QU Vul $K$ band light curve superimposed on a binned $K$ band photometric curve.

Table 4. Best model fit parameters for QU Vul.

\begin{tabular}{ll}
\hline \hline Free parameter & Model value \\
\hline WD mass & $0.82 M_{\odot}$ \\
Secondary star mass & $0.22 M_{\odot}$ \\
WD temp & $65000 \mathrm{~K}$ \\
Secondary star pole temp & $3500 \mathrm{~K}$ \\
Mass transfer rate & $7.0 \times 10^{-9} M_{\odot} \mathrm{yr}^{-1}$ \\
Disc outer dia & $0.7 L_{1}$ \\
Disc inner dia & $0.3 L_{1}$ \\
Disc opening angle & $3^{\circ}$ \\
Inclination & $76^{\circ}$ \\
Gravitational darkening coefficient & 0.08 \\
Limb darkening coefficient & 0.4 \\
Orbital period & $9648 \mathrm{~s}$ \\
\hline Derived parameter & Model value \\
\hline Binary separation & $0.98 R_{\odot}$ \\
RL1 separation ratio & 0.63 \\
Disc radius & $0.44 R_{\odot}$ \\
Disc height & $2.2 \times 10^{-3} R_{\odot}$ \\
Outer disc temp & $59000 \mathrm{~K}$ \\
Secondary star radius & $0.36 R_{\odot}$ \\
White dwarf radius & $9.5 \times 10^{-3} R_{\odot}$ \\
Reduced chi-squared & 4.9 \\
\hline
\end{tabular}

luminosity would indicate that the secondary star is being irradiated by $8.4 L_{\odot}$. However for a given value of the distance parameter (set in the model to the lowest estimated value) the model calculates a value for the mass transfer rate which is required to match the disc luminosity. Thus, for example, a $5 \%$ increase in the distance to the system can be traded off with a $50 \%$ increase in mass transfer rate in order to achieve the same $\chi^{2}$ goodness of fit. Since the WD is relatively hot, it is irradiating the disc and thus accretion is not the only source of disc luminosity. Since the model assumes all the disc luminosity is due to accretion the accretion rate is overestimated. The modelled value of irradiating luminosity of $1.4 L_{\odot}$ is in reasonable agreement with the values obtained from the flux analysis.

\subsection{Per irradiation}

Since the $K$ band light curve shows the presence of a hot spot on the disc, and this would give rise to an erroneous value for the flux ratio of the system, we took photometric data from Shafter \& Abbott (1989) who observed V Per in a broad band " $B V R$ "

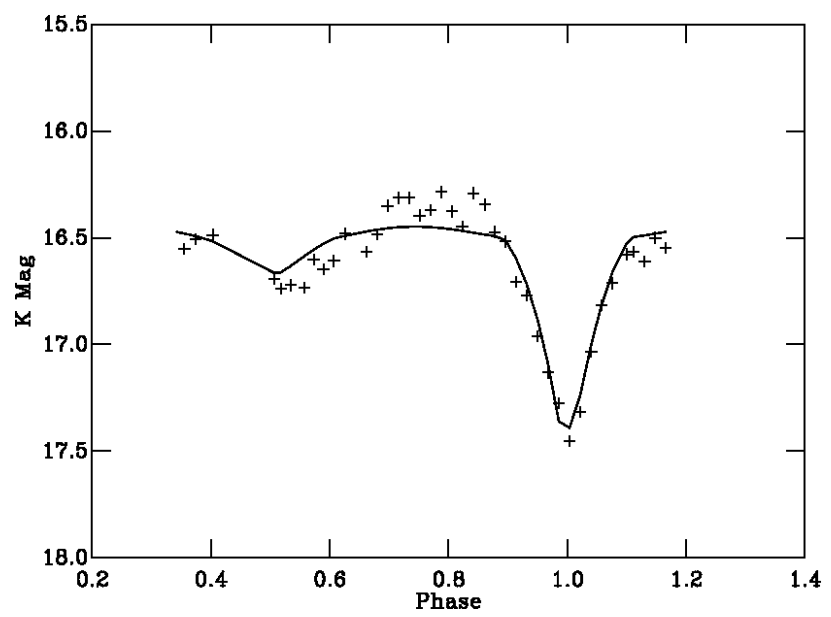

Fig. 11. Best fit model for the V Per $K$ band light curve superimposed on a binned $K$ band photometric light curve.

filter which transmits from approximately $4000 \AA$ to $7000 \AA$. Flux ratios were calculated from values of the relative intensity at fiducial points on three light curves, giving a mean flux ratio of 1.19 with an uncertainty of 0.02 implying that V Per has an irradiating luminosity of $0.043_{-0.001}^{+0.002} L_{\odot}$.

Modelling for V Per was carried out in a similar manner to that used above for QU Vul. It is found that a range of close fits to the model for V Per could be obtained for reasonable values of outer disc radius, disc opening angle and white dwarf temperature. However since the model does not take into account the presence of the hot spot, the best reduced chi-squared fit that can be made between the model and the light curve for V Per is of the order of 6.3. Improving the fit to the primary eclipse could only be made at the expense of a poorer fit to the secondary eclipse and vice versa.

The best fit model required a relatively a thick accretion disc of $7^{\circ}$ semi-opening angle, with the temperature of the hot white dwarf being $10000 \mathrm{~K}$. At a chosen value of white dwarf mass $0.84 M_{\odot}$, the white dwarf radiates at approximately $0.0007 L_{\odot}$. This value sets a lower limit on the heating effect. This value is in good agreement with the lower end of the temperature range for the white dwarf in V Per in BVRI modelling undertaken by Shafter \& Misselt (2006).

As the opening angle of the disc controls the shading of the inner face of the secondary star, increases in the irradiating flux can be traded off with the disc-opening angle. It was found that a reasonable fit, having a reduced $\chi^{2}$ of 6.34 , could be obtained with an opening angle of $9^{\circ}$ with a a white dwarf temperature of $30000 \mathrm{~K}$. At this temperature the WD luminosity is in the region of $0.04 L_{\odot}$ which is consistent with the upper limit of the WD temperature modelled by Shafter \& Misselt and is also consistent with the value of the irradiating luminosity determined by the flux ratio method. The results of the best fit are shown in Fig. 11 and Table 5. The model for $\mathrm{V}$ Per requires a mass transfer rate of $1.0 \times 10^{-9} M_{\odot} \mathrm{yr}^{-1}$ and this is of a similar orderof-magnitude to the lower values of mass transfer rate presented in Shafter \& Misselt (2006) and Shafter et al. (1986), to the estimate of $2.0 \times 10^{-9} M_{\odot} \mathrm{yr}^{-1}$ for the critical mass tranfer rate required to keep V Per's disc stable. For a mass transfer rate of $1.0 \times 10^{-9} M_{\odot} \mathrm{yr}^{-1}$ this would be expected to give rise to an accretion luminosity in the region of $1.2 L_{\odot}$ and yet we do not see this. It would appear the disc in V Per hides the majority of the accretion radiation from the secondary. With such a low apparent irradiation the white dawf is not heating the secondary so it 
Table 5. Best model fit parameters for V Per.

\begin{tabular}{ll}
\hline \hline Free parameter & Model value \\
\hline WD mass & $0.84 M_{\odot}$ \\
Secondary star mass & $0.21 M_{\odot}$ \\
WD temp & $10000 \mathrm{~K}$ \\
Secondary star pole temp & $2900 \mathrm{~K}$ \\
Mass transfer rate & $1.0 \times 10^{-9} M_{\odot} \mathrm{yr}^{-1}$ \\
Disc outer dia & $0.6 L_{1}$ \\
Disc inner dia & $0.0 L_{1}$ \\
Disc opening angle & $7^{\circ}$ \\
Inclination & $86^{\circ}$ \\
Gravitational darkening coefficient & 0.08 \\
Limb darkening coefficient & 0.4 \\
Orbital period & $9252 \mathrm{~s}$ \\
\hline Derived parameter & Model value \\
\hline Binary separation & $0.99 R_{\odot}$ \\
RL1 separation ratio & 0.63 \\
Disc radius & $0.37 R_{\odot}$ \\
Disc height & $4.6 \times 10^{-2} R_{\odot}$ \\
Outer disc temp & $5100 \mathrm{~K}$ \\
Secondary star radius & $0.36 R_{\odot}$ \\
White dwarf radius & $9.4 \times 10^{-3} R_{\odot}$ \\
Reduced chis-squared & 6.3 \\
\hline
\end{tabular}

must have cooled. The flux ratio method confirms that heating of the inner Lagrangian face of the secondary star by a hot white dwarf companion is not occurring in V Per.

\subsection{DD Cir (Nova Cir 1999)}

Flux ratios were measured at six fiducial phases on four $V$ band light curves of DD Cir obtained by Wouldt \& Warner (2003). This gave a mean flux ratio of 2.284 with a standard deviation of 0.049 . This is equivalent to a mean irradiating luminosity of $0.7_{-0.1}^{+1.0} L_{\odot}$.

Fiducial data points were taken from the same light curves and the system was modelled using their values for the angle of inclination of the system, disc radius and estimated mass of the primary and secondary star. The results of the modelling show that it is possible to obtain a good agreement with their light curve if the secondary star is irradiated by $1.04 L_{\odot}$ which compares reasonably with the flux ratio value of irradiation.

\subsection{DN Gem (Nova Gem 1912)}

Data were taken from the $I$ band observations of DN Gem, observed between 1995 and 1998, by Retter et al. (1999) and a single light curve produced from a least squares fit to a 6th order polynomial. This gave a fit of $R^{2}=0.94$. Flux ratios were then calculated at the fiducial points on the resulting light curve giving a flux ratio of 2.07. Converting the flux ratio to irradiating luminosity gave a mean value for DN Gem as $1.12 L_{\odot}$.

Retter et al modelled DN Gem and showed that the sinusoidal modulation in its light curve arose from an irradiated secondary star. Our modelling code was used with data taken from Retter's model to calculate the WD temperature and identify the extent of the irradiation from the white dwarf. Setting the disc opening angle to a minimum, the white dwarf temperature was adjusted to provide the best fit, the results of the modelling give the minimum irradiation as approximately $1.3 L_{\odot}$ consistent with the figure derived from flux ratio analysis.

\subsection{WY Sge (Nova Sge 1783)}

Data taken from the $K$ band binned observations of WY Sge by Somers et al. (1996) were treated in a similar maner to that described above for DN Gem and regression analysis using a 6th order polynomial $\left(R^{2}\right.$ fit $\left.=0.96\right)$ resulted in a flux ratio of 1.5 at the fiducial points. This predicts the mean irradiating luminosity of WY Sge to be $0.50 L_{\odot}$.

The infrared modelling data of Somers et al. (1996) indicate that the secondary star is heated by a central irradiating source of $0.56 L_{\odot}$. This is again consistent with the level of irradiation predicted by the flux ratio method.

\subsection{V1432 Aql}

Data points for the near asynchronous polar V1432 Aql were taken from a mean light curve drawn through the stepped light curve presented in Friedrich et al. (1996). This gave a flux ratio of 1.89 and an irradiating luminosity of $0.18 L_{\odot}$ which compares well with the estimate of Schmidt \& Stockman (2001) who determined the white dwarf in V1432 Aql to have a luminosity of between 0.2 to $0.5 L_{\odot}$. The exact date of the nova is unknown but Schmidt \& Stockman suggest that V1432 Aql underwent a nova eruption between 75 to 150 yrs ago.

\section{Change in irradiation with time since the nova explosion}

Our flux ratio analysis estimates the degree of irradiation of the secondary irrespective of whether the source of the irradiation is from the boundary layer or from a hot white dwarf. It may be possible to further discriminate between these sources if we had reliable estimates of the accretion rate for our sample of CVs. Bearing in mind the effect of irradiation on the acretion disc, such mass transfer rates would, unfortunately, need to be derived by a method which is not simply dependent on the luminosity of the disc. Further clarity can, however, be obtained if, instead, we look at the change in irradiation with time since the nova explosion.

\subsection{Short term evolution and decline in mass transfer rate}

The results of the flux ratio analysis for our sample of CVs show that, even a few years after the nova explosion, the secondary stars appear to be irradiated very little. In QU Vul, for example, the radiating luminosity emmitted by the white dwarf is of the order of $1.4 L_{\odot}$. Since the red dwarf subtends a solid angle equivalent to $1 \%$ of the sky as seen from the white dwarf, and in addition, the disc shadows a portion of the secondary star, it means that the secondary only receives of the order of $0.01 L_{\odot}$.

Since the secondary in QU Vul is shadowed by the accretion disc the inner Lagrangian point may also be effectively shielded by the disc. Meridonal circulation (Kippenhahn \& Thomas 1979; Sarna 1990), however, has been proposed to provide a mechanism for energy transport from the illuminated regions towards the region near the $\mathrm{L} 1$ point thereby overcoming these shielding effects and allowing such irradiated secondaries to increase mass transfer.

This then begs the question - is this level of irradiation sufficient to drive mass transfer in CVs during the period in which the nova cools and hence affect short term evolution?

The possibility of short-term irradiation-induced mass transfer in some dwarf novae, whereby radiation from the accretion disc during superoutburst results in an enhancement of the mass 
transfer rate, has been used by Schreiber et al. (2004) to explain the light curves of SU UMa stars and by Schreiber \& Lasota (2007) to solve the distance problem associated with SS Cyg. Recent theoretical calculations by Viallet \& Hameury (2007) indicate that such effects, however, are thought to only last for 10 to 20 orbital periods and are unlikley to be able to account for the duration of long outbursts.

Long term irradiation effects, on the thermal timescale of the convective envelope of the donor star, involving the existence of limit cycles have been extensively investigated by many authors. King (1995) showed that weak anisotropic irradiation of the secondary star can drive mass transfer cycles in CVs where the donor star has a reasonable base mass transfer rate and is almost fully convective. These cycles are based on radius variation of the secondary star resulting from irradiation blocking the secondary's own interior heat flux leaving the star. The system oscillates between high states, in which the irradiation causes slow expansion of the secondary which drives an elevated mass transfer rate, followed by low states in which the secondary star contracts.

Ritter et al. (2000) showed that optimum irradiation is achieved in such cycles when as large a fraction as possible of the secondary's surface is irradiated and, as we have for QU Vul, the irradiating flux is of the same order of magnitude as the secondary star flux (once the effective irradiating flux exceeds a value of the order of the secondary flux no more luminosity can be blocked in).

However their work shows that the timescale on which the irradiated star initially expands is of the order of the thermal time scale of the convective envelope $\left(\approx 10^{8}\right.$ years $)$. Therefore its is unlikely that the degree of irradiation we have seen in our sample of CVs could play a significant part in driving mass transfer during the relative short time scales involved with irradiation from the cooling hot WD after a nova eruption. It would be useful if this could be theoretically confirmed.

\subsection{Short term evolution and decline in irradiation}

If the irradiating luminosity is not driving the mass transfer rate, then it is hard to explain the fading of novae in the years after outburst as a decline in accretion luminosity. To investigate this further we examined the change in irradiation with time. Conversion of the flux ratios to irradiating luminosity and plotting the irradiating luminosity as a function of time since the nova explosion, for the novae discussed in the text, shows there may be a fall in luminosity with time, as would be expected (Fig. 12). However as the nova ages there appears to be a marked degree of scatter.

In order to find the rate of decline of luminosity we performed a linear regression analysis on the data. Since there is a large degree of scatter in the data, we formulated the statistical null hypothesis that the true slope of the regression line was zero against the alternative hypothesis that the slope was not zero, for varying levels of probability (i.e. we tested whether there was a linear relationship between the two variables). The results of the t-test indicate that the slope of the linear regression line is not significant at the $1 \sigma$ probability level.

However, for what follows, it is crucial we establish the range of possible slopes which may exist within a our data set. To do this we undertook a non-parametric bootstrap analysis with replacement.This was caried out by taking the six data points, removing one point and randomly replacing it with one of the other points, similarly with the next point, and so on until all 6 data points had been removed and replaced. The slope of the

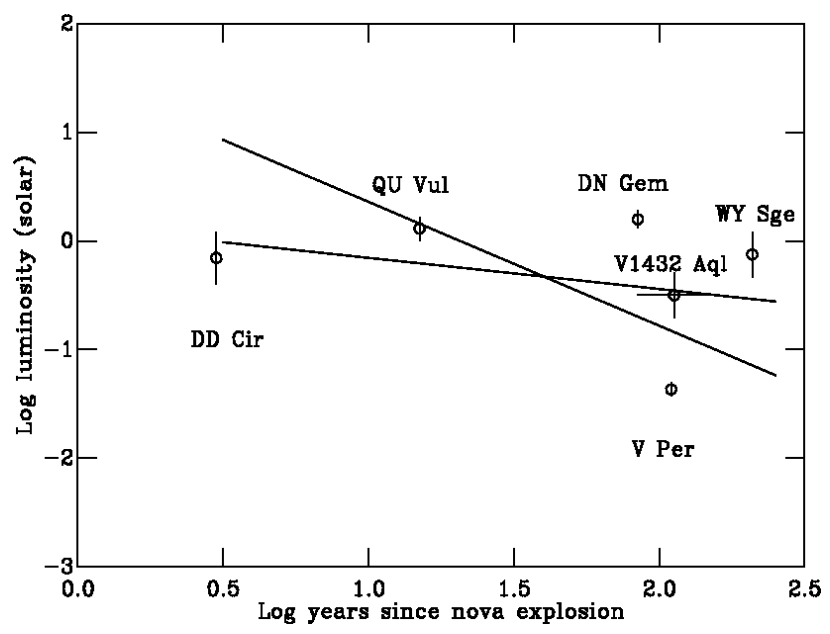

Fig. 12. Irradiating luminosity as a function of time since the nova explosion for the novae discussed in the text. The line with the steeper gradient is the theoretical WD cooling rate $\left(L \propto t^{-1.14}\right)$, while the other line is the least squares fit to the data $\left(L \propto t^{-0.35}\right)$.

resulting irradiation time plot was found through linear regression, and the process repeated 1000 times. This gave a population mean of -0.392 and population standard deviation of 0.685 . Applying confidence limits at the $1 \sigma$ level sets the limits of the slope to be +0.3 and -1.1 .

The rate of decline of irradiating luminosity for the six chosen CVs, obtained from flux ratio analysis, based on the linear regression, gives a decline in magnitude of 13.5 millimags per year (i.e. slope of -0.351 on the log irradiation-log time plot). Although we cannot be absolutely sure that it is just irradiation from the WD or whether it is WD irradiation together with some accretion irradiation, we can see no reason why the mass transfer rate should be decreasing and the results of the flux ratio technique strongly suggest that this rate of decline appears to be based on WD irradiation alone and not involving any irradiation as a result of mass transfer.

This result corresponds closely to Duerbecks (1992) mean value of 10.0 plus or minus 3 millimags per year for nine old novae, half a century after outburst which gives rise to slopes between -0.185 and -0.344 . Vogts (1990) result of 15 to 27 millimags per year gives rise to slopes of -0.396 and -0.71 . Both Duerbeck's and Vogt's results sit within the $68 \%$ confidence limits of +0.3 and -1.1 obtained from the bootstrap results.

Vogt's dataset is a cross-sectional study of 97 novae in which the rate of change in brightness, after the outburst, was determined from eruption amplitudes (difference in brightness between that of outburst and that at quiesence) and decay times (time taken for the brightness to decrease 3 mag from outburst maximum). Duerbeck's, on the other hand, is a longitudinal study based on visual observations and photoelectric measurements of old novae, made over timescales of up to 80 years, following their decline. Given the clarity of Duerbecks results its is suprising that Vogt's result (based on 10 times as many stars) is not stronger. Both Vogt's and Duerbeck's techniques would be expected to give rise to a better estimate of the rate of decline in luminosity than our six objects. Our technique, on the otherhand, has higher precision in determining the irradiation as it simply measures the irradiation of the secondary rather than total luminosity of the CVs, yet there is still a large amount of scatter in the data which indicates that the white dwarf may well be heated to significantly different temperatures in each nova increasing the 
uncertainty of our estimate. This would also explain the greater degree of scatter in Vogt's results than that seen in Duerbeck's.

Since the results of Vogt and Duerbeck lie within the bootstrap $68 \%$ confidence limits of our bootsrap model, as does the slope of theoretical WD cooling rate of $\left(L \propto t^{-1.14}\right.$, we make the case that the decline seen by both Vogt and Duerbeck is not a decline in mass transfer rate, but in irradiation from the WD. These results are also consistent with Schreiber \& Gänsicke's (2001) calculations who find that the visual brightness of post novae gradually decreases after the nova explosion as a result of decreasing irradiation of the disc from the continuously cooling white dwarf.

\section{Conclusions}

The light curves of QU Vul show the presence of maxima in the $K$ band which are displaced from phase 0.25 to the 0.5 phase position. This completely fills in the secondary eclipse, as would be expected from heating of the inner face of the secondary star by radiation from a hot primary. Nova V Per on the other hand shows evidence for the presence of a bright spot on the accretion disc and it would appear that the heating of the inner face is not occurring.

For the small number of CVs sampled, the WD irradiating luminosity derived by the flux ratio technique has been shown to give reasonably good agreement with that found by model fitting the lightcurve.

The observed decline in irradation with time we see in our sample has been shown to be consistent with that produced by the cooling of the hot white dwarf. Our bootstrap analysis shows that Duerbeck's and Vogt's results are also consistent with this. Since current theories of irradiation induced mass transfer do not support the idea that it can influence the short term evolution of CVs, and our results of the decline in irradiation with time are able to be explained by the cooling effect, we see no further need to introduce additional sources of irradiation i.e. decreasing irradiation from the boundary layer.

Although we cannot rule out the effects of mass transfer rate on the irradiation of the secondary, the results of the flux ratio analysis on the objects examined indicate that the decline seen in old novae is consistent with the idea that it is the long-term decrease in irradiation with time since the nova explosions occurred, not mass transfer decline, which we see in old novae. Despite the precision of the method we see a considerable degree of scatter in the data which is possibly caused by the different temperatures reached in the nova explosion, which explains why Duerbeck's relative experiment works with a much smaller number of stars than Vogt's absolute one. Despite the scatter, this decline is consistent with long term simulations of post nova showing their decrease in optical brightness is associated with decreasing disc irradiation from the cooling white dwarf.

Acknowledgements. The infrared observations were taken with the 3.8m Cassegrain United Kingdom Infra Telescope (UKIRT) at Mauna Kea, Hawaii. The UK Infrared Telescope is operated by the Joint Astronomy Centre on behalf of the UK Particle Physics and Astronomy Research Council.
This publication makes use of data products from the Two Micron All Sky Survey, which is a joint project of the University of Massachusetts and the Infrared Processing and Analysis Centre/California Institute of Technology, funded by the National Aeronautics and Space Administration and the National Science Foundation.

\section{References}

Al-Naimiy, H. M. 1978, Ap\&SS, 53, 181

Andrea, J., Drechsel, H., \& Starrfield, S. 1994, A\&A, 291, 869

Bergner, Y. K., Bondarenko, S. I., Miroshnichenko, A. S., et al. 1985, Astron. Tsirk., 1374

Bochkarev, N. G., Karitskaya, E. A., \& Shakura, N. I. 1979, AZh, 56, 16B

Cutri, R. M., Skrutskie, M. F., van Dyk, S., et al. 2003, The IRSA 2MASS AllSky Point Source Catalog, NASA/IPAC Infrared Science Archive

Della Valle, M., Gilmozzi, R., Bianchini, R., \& Esenoglu, H. 1997, A\&A, 325, 1156

Downes, R. A. 1986, ApJ, 307, 286

Downes, R. A., \& Duerbeck, H. W. 2000, AJ, 120, 2007

Duerbeck, H. W. 1992, MNRAS, 258, 629

Friedrich, S., Staubert, R., Lamer, G., et al. 1996, A\&A, 306, 860

Gehrz, R. D., Grasdalen, G. L., \& Hackwell, J. A. 1985, ApJ, 298, L47

Hawarden, T. G., Leggett, S. K., Letawsky, M. B., Ballantyne, D. R., \& Casli, M. M. 2001, MNRAS, 325, 563

Hill, G. 1979, DAO, 15, 297

Hoard, D. W., Szkody, P., Honeycutt, R. K., et al. 2000, PASP, 112, 778, 1595

Ioannou, Z., Naylor, T., Welsh, et al. 1999, MNRAS, 310, 2

King, A. R., Frank, J., Kolb, U., \& Ritter, H. 1995, ApJ, 444, L40

Kippenhahn, R., \& Thomas, H.-C. 1979, A\&A, 75, 281

Kovetz, A., Prialnik, D., \& Shara, M. 1988, ApJ, 325, 828

Mochnacki, S. W., \& Doughty, N. A. 1972, MNRAS, 156, 52

Naylor, T. 1998, MNRAS, 296, 339

Paneck, R. J., \& Eaton, J. A. 1982, ApJ, 258, 572

Patterson, J. 1984, ApJS, 54, 443

Prialnik, D. 1986, ApJ, 310, 222

Retter, A., Leibwitz, E. M., \& Naylor, T. 1999, MNRAS, 308, 140

Ritter, H., Politano, M., Livio, M., \& Webbink, R. F. 1991, ApJ, 276, 177

Ritter, H., Zhang, Z.-Y., \& Kolb, U. 2000, A\&A, 360, 969

Saizar, P., Starrfield, S., Ferland, et al. 1992, ApJ, 398

Sarna, M. J. 1990, A\&A, 239, 163

Schmidt, G. D., \& Stockman, H. S. 2001, ApJ, 548, 410s

Shabaz, T., Naylor, T., \& Charles, P. A. 1993, MNRAS, 265, 655

Shafter, A. W. 1997, ApJ, 487, 226

Shafter, A. W., \& Abbott, T. M. C. 1989, ApJ, 339, L75

Shafter, A. W., \& Misselt, K. A. 2006, ApJ, 644, 1104

Shafter, A. W., Wheeler, J. C., \& Cannizzo, J. K. 1986, ApJ, 305, 261

Shafter, A. W., Misselt, K. A., Szkody, P., et al. 1995, ApJ, 448, L33

Shara, M. M., Livio, M., Moffat, A. F. J., \& Orio, M. 1986, ApJ, 311, 163

Schreiber, M. R., \& Gänsicke, B. T. 2001, A\&A, 375, 937

Schreiber, M. R., \& Lasota, J.-P. 2007, A\&A, 473, 897

Schreiber, M. R., Hameury, J.-M., \& Lasota, J.-P. 2004, A\&A, 427, 621

Shin, J. Y., Gehrz, R. D., Jones, T. J., et al. 1998, AJ, 116, 1966

Somers, M. W., \& Naylor, T. 1999, A\&A, 352, 563

Somers, M. W., Mukai, K., \& Naylor, T. 1996, MNRAS, 278, 845

Taylor, A. R., Hjellming, R. M., Seaquist, E. R., \& Gehrz, R. D. 1998, Nature, 335,235

Tjemkes, S. A., Zuiderwijk, E. J., \& van Paradijs, J. 1986, A\&A, 60, 471

Warner, B. 1995, Cataclysmic Variable Stars (Cambridge: Cambridge University Press)

Webbink, R. F. 1990, Accretion-Powered Compact Binaries, ed. C. W. Mauche (Cambridge: Cambridge University Press)

Weight, A., Evans, A., Naylor, T., Wood, J. H., \& Bode, M. F. 1994, MNRAS, 266,761

Wilson, R. E., \& Devinney, E. J. 1979, ApJ, 166, 605

Woudt, P. A., \& Warner, B. 2003, MNRAS, 340, 1011

Viallet, M., \& Hameury, J.-M. 2007, A\&A, 475, 597

Vogt, N. 1990, ApJ, 356, 609 